\title{
SUPERLATTICE PHOTOCATHODES FOR ACCELERATOR-BASED POLARIZED ELECTRON SOURCE APPLICATIONS*
}

\author{
J. E. Clendenin", T. Maruyama, G. A. Mulhollan \\ Stanford Linear Accelerator Center, Stanford, CA 94309, USA \\ A. V. Subashiev, Yu. A. Mamaev, Yu. P. Yashin \\ St. Petersburg Technical University, St. Petersburg, 195251, Russia
}

\begin{abstract}
A major improvement in the performance of the SLC was achieved with the introduction of thin strained-layer semiconductor crystals. After some optimization, polarizations of $75-85 \%$ became standard with lifetimes that were equal to or better than that of thick unstrained crystals. Other accelerators of polarized electrons, generally operating with a much higher duty factor, have now successfully utilized similar photocathodes. For future colliders, the principal remaining problem is the limit on the total charge that can be extracted in a time scale of 10 to $100 \mathrm{~ns}$. In addition, higher polarization is critical for exploring new physics, especially supersymmetry. However, it appears that strained-layer crystals have reached the limit of their optimization. Today strained superlattice crystals are the most promising candidates for better performance. The individual layers of the superlattice can be designed to be below the critical thickness for strain relaxation, thus in principle improving the polarization. Thin layers also promote high electron conduction to the surface. In addition the potential barriers at the surface for both emission of conduction-band electrons to vacuum and for tunneling of valence-band holes to the surface can be significantly less than for single strained-layer crystals, thus enhancing both the yield at any intensity and also decreasing the limitations on the total charge. The inviting properties of the recently developed AlInGaAs/GaAs strained superlattice with minimal barriers in the conduction band are discussed in detail.
\end{abstract}

\author{
Contributed to the \\ 1999 Particle Accelerator Conference \\ New York City, New York \\ March 29 - April 2, 1999
}

\footnotetext{
"Work supported by the U.S. Department of Energy contract DE-AC03-76SF00515, the U.S. Civilian Research and Development Foundation Award No. RP1-351, the Russian State Program Project 2.6.99, and the Russian State Programs "Physics of Solid State Nanostructures" under grant 97-1091.

\# Email: clen@slac.stanford.edu
} 


\title{
SUPERLATTICE PHOTOCATHODES FOR ACCELERATOR-BASED POLARIZED ELECTRON SOURCE APPLICATIONS*
}

\author{
J. E. Clendenin ${ }^{\#}$, T. Maruyama, G. A. Mulhollan, SLAC, Stanford, CA \\ Yu. A. Mamaev, A. V. Subashiev, Yu. P. Yashin, SPTU, St. Petersburg, Russia
}

\begin{abstract}
A major improvement in the performance of the SLC was achieved with the introduction of thin strained-layer semiconductor crystals. After some optimization, polarizations of $75-85 \%$ became standard with lifetimes that were equal to or better than that of thick unstrained crystals. Other accelerators of polarized electrons, generally operating with a much higher duty factor, have now successfully utilized similar photocathodes. For future colliders, the principal remaining problem is the limit on the total charge that can be extracted in a time scale of 10 to $100 \mathrm{~ns}$. In addition, higher polarization is critical for exploring new physics, especially supersymmetry. However, it appears that strained-layer crystals have reached the limit of their optimization. Today strained superlattice crystals are the most promising candidates for better performance. The individual layers of the superlattice can be designed to be below the critical thickness for strain relaxation, thus in principle improving the polarization. Thin layers also promote high electron conduction to the surface. In addition the potential barriers at the surface for both emission of conductionband electrons to vacuum and for tunneling of valenceband holes to the surface can be significantly less than for single strained-layer crystals, thus enhancing both the yield at any intensity and also decreasing the limitations on the total charge. The inviting properties of the recently developed AlInGaAs/GaAs strained superlattice with minimal barriers in the conduction band are discussed in detail.
\end{abstract}

\section{INTRODUCTION}

The polarization of electrons extracted from III-V semiconductor crystals has a theoretical upper limit of $50 \%$. In 1991 it was first demonstrated that growing a thin crystalline layer on a substrate having a slightly different lattice constant could raise this limit [1]. The lattice mismatch introduces a strain in the epilayer that removes the degeneracy of the heavy-hole (hh) and lighthole (lh) valence bands. Polarizations on the order of $80 \%$ are routinely achieved from these strained-layer cathodes, which simultaneously exhibit quantum yields, $Y$, of $>0.1 \%$. Such a cathode, consisting of $100-\mathrm{nm}$ of GaAs grown on a GaAsP substrate, became the standard for the SLC polarized electron source, eventually accumulating $\sim 20,000$ hours of operating time for the SLC program, making possible the single-most precise measurement of the electro-weak mixing angle, $\sin ^{2} \theta_{W}^{e f f}$.
Future colliders will require both higher polarization and more charge. Higher polarization will not only increase the effective luminosity of a collider, but if $\geq 95 \%$ will also significantly increase the physics reach, especially in the exploration of supersymmetry [2]. For JLC/NLC, the required charge per 100-ns macropulse is $\sim 300 \mathrm{nC}$ at the gun, which is an order of magnitude greater than produced for SLC. The problem is that the maximum steady-state current in a long macropulse that can be produced from an SLC-type cathode is on the order of $0.2 \mathrm{~A} \mathrm{~cm}^{-2}$, which would require an impractical cathode diameter of $4 \mathrm{~cm}$ to meet the collider charge requirement. The maximum current of an SLC-type cathode can probably be increased by an order of magnitude simply by increasing the dopant density from the mid $10^{18} \mathrm{~cm}^{-3}$ range to the low $10^{19} \mathrm{~cm}^{-3}$ range. However, both experimental and theoretical efforts to date point to a limitation of the polarization of the strained-layer cathode at the $85-90 \%$ level [2].

A major source of depolarization for the strained-layer cathodes is the relaxation of the strain as the distance from the heterojunction increases beyond the critical thickness, $\mathrm{h}_{\mathrm{c}}$, for maintaining perfect strain, thus decreasing the average hh-lh separation. Typically $h_{c}$ is $\sim 10 \mathrm{~nm}$, whereas the optimum epilayer thickness to maintain both high polarization and high charge is $\sim 100 \mathrm{~nm}$. This problem is overcome in principle using the superlattice (SL) structure, in which the hh-lh degeneracy is removed by the localization of holes in quantum wells. Since the overall critical thickness, Hc, for a short-period SL can be considerably larger than $h_{c}$, the total thickness of the SL can be on the order of $100 \mathrm{~nm}$ without relaxation of the strain.

SL structures were shown to produce $>50 \%$ polarization in 1991 soon after the first strained-layer results, but the path to simultaneously high polarization and high $Y$ has taken longer. Recently however, polarizations approaching $90 \%$ with $Y$ within an order of magnitude of that of the SLC cathodes have appeared $[3,4]$.

\section{SUPERLATTICE WITH ZERO CONDUCTION BAND OFFSET}

In previously used $\mathrm{Al}_{\mathrm{x}} \mathrm{Ga}_{1-\mathrm{x}} \mathrm{As} / \mathrm{GaAs}, \mathrm{In}_{\mathrm{x}} \mathrm{Ga}_{1-\mathrm{x}} \mathrm{As} / \mathrm{GaAs}$ and $\mathrm{Al}_{\mathrm{x}} \mathrm{Ga}_{1-\mathrm{x}} \mathrm{As} / \mathrm{In}_{\mathrm{y}} \mathrm{Ga}_{1-\mathrm{y}} \mathrm{As} \mathrm{SL}$, the hole miniband splitting was accompanied by the building of high barriers for electrons as a result of the high value of the ratio of the conduction-band offset $\Delta E_{c}$ to the valence-band offset $\Delta \mathrm{E}_{\mathrm{v}}$ $\left(\Delta E_{c} / \Delta E_{v} \approx 2\right)$ at the heterointerface. The necessity for the electrons to tunnel through the SL barriers results in a lower electron diffusion rate along the SL axis

\footnotetext{
*Work supported by the U.S. Department of Energy contract DE-AC03-76SF00515, the U.S. Civilian Research and Development Foundation Award No. RP1-351, the Russian State Program Project 2.6.99, and the Russian State Programs "Physics of Solid State Nanostructures" under grant 97-1091.

\#Email: clen@slac.stanford.edu
} 
accompanied by growth of the spin relaxation rate. Therefore the spin diffusion length restricts the thickness of the active layer for these structures.

The main advantage of the $\mathrm{Al}_{\mathrm{x}} \mathrm{In}_{\mathrm{y}} \mathrm{Ga}_{1-\mathrm{x}-\mathrm{y}} \mathrm{As} / \mathrm{GaAs} \mathrm{SL}$ proposed here comes from the band line-up between the semiconductor layers of the SL. The Al content determines the formation of a barrier in the conduction band, while adding In leads to conduction band lowering, so the conduction band offset can be completely compensated by appropriate choice of $\mathrm{x}$ and $\mathrm{y}$, while barriers for the holes remain uncompensated. Therefore the use of superlattices with the optimized quaternary alloy composition can provide a high vertical electron mobility and simultaneously a small spin relaxation rate while also maintaining a sufficiently large valence-band splitting.

We have studied an $\mathrm{Al}_{0.20} \mathrm{In}_{0.18} \mathrm{Ga}_{0.62} \mathrm{As} / \mathrm{GaAs} \mathrm{SL}$ consisting of 17 pairs of GaAs (4 nm) and AlInGaAs (4 $\mathrm{nm})$ Be doped with density $4 \times 10^{17} \mathrm{~cm}^{-3}$ except the top layer, GaAs, with $4 \times 10^{18} \mathrm{~cm}^{-3}$. The dopant density was maintained low in the bulk to minimize depolarization but high at the surface to increase the negative affinity. The sample was grown by molecular beam epitaxy (MBE) by the Ioffe Physico-Technical Institute in St. Petersburg [4]. A protective As coating was added as the final step.

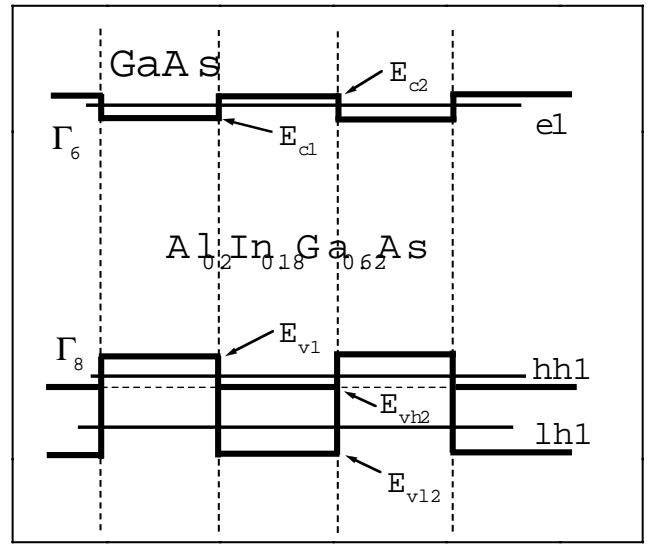

Fig. 1. Energy band diagram of $\mathrm{Al}_{\mathrm{x}} \mathrm{In}_{\mathrm{y}} \mathrm{Ga}_{1-\mathrm{x}-\mathrm{y}} \mathrm{As} / \mathrm{GaAs} \mathrm{SL}$. The minibands (thin lines) are identified by notation e1 and hh1, lh1 for electrons and holes respectively.

The miniband spectrum of the SL is determinedby the band offsets at the heterointerfaces. In the case of a heterointerface with lattice matched ternary solid solution (e.g., $\mathrm{Al}_{\mathrm{x}} \mathrm{Ga}_{1-\mathrm{x}} \mathrm{As} / \mathrm{GaAs}$ ) the conduction-band offset ratio, $Q_{c l}$, is definedas $Q_{c 1}=\Delta E_{c}(x) / \Delta E_{g 1}(x)$, where $\Delta E_{g 1}$ is the differencein the band gaps of the contacting crystals so that the valence band offset ratio, $Q_{v}$, is $Q_{v}=1-Q_{c}$. For an $\mathrm{In}_{\mathrm{y}} \mathrm{Ga}_{1-\mathrm{y}} \mathrm{As} / \mathrm{GaAs}$ interface the offset is modified by the strain distribution in the contacting layers. For the structure with a thin $\operatorname{In}_{\mathrm{y}} \mathrm{Ga}_{1-\mathrm{y}}$ As layer grown on a thick GaAs substrate all the strain is assumed to accumulate in the InGaAs layer. For this case, $\Delta E_{c}$ is given by:

$$
\Delta E_{c}(y)=Q_{c 2, \text { def }} \times\left(\Delta E_{g 2}(y)+\delta E_{g 2}^{\text {def }}(y)\right),
$$

where $Q_{c 2 \text {,def }}$ is the conduction-bandoffset ratio for the strained $\operatorname{In}_{\mathrm{y}} \mathrm{Ga}_{1-\mathrm{y}} \mathrm{As}$ layer, while $\delta E_{g 2}^{d e f}(y)$ is the change of the band gap induced by strain that can be expressed through interpolated deformation potentials, elastic constants, and the lattice constant mismatch. For the case of the $\mathrm{Al}_{\mathrm{x}} \mathrm{In}_{\mathrm{y}} \mathrm{Ga}_{1-\mathrm{x}-\mathrm{y}} \mathrm{As} / \mathrm{GaAs} \mathrm{SL}$, a linear interpolation between the values for $\mathrm{Al}_{\mathrm{x}} \mathrm{Ga}_{1-\mathrm{x}} \mathrm{As} / \mathrm{GaAs}$ and $\mathrm{In}_{\mathrm{y}} \mathrm{Ga}_{1-}$ ${ }_{\mathrm{y}} \mathrm{As} / \mathrm{GaAs}$ interfaces should be valid for small $\mathrm{x}$ and $\mathrm{y}$. We have found that interpolation starting with the unstrained value of the band offset for the $\operatorname{In}_{\mathrm{y}} \mathrm{Ga}_{1-\mathrm{y}} \mathrm{As} / \mathrm{GaAs}$ heterointerfacegives band-gap values that are far from the experimental data obtained from emission spectra. Using the band offset value for the strained $\operatorname{In}_{\mathrm{y}} \mathrm{Ga}_{1-\mathrm{y}} \mathrm{As} / \mathrm{GaAs}$ interface, we get:

$$
\Delta E_{c}(x, y)=Q_{c 1} \times \Delta E_{g 1}(x)+Q_{c 2, \text { def }} \times\left(\Delta E_{g 2}(y)+\delta E_{g 2}^{\text {def }}(y)\right) .
$$

The schematic of the position of the band edges for $\mathrm{x}=0.20, \mathrm{y}=0.18$ is shown in Fig. 1 . Taking $Q_{c l}=0.66$ and $Q_{c 2, d e f}=0.7$, we have found that the conduction band offset appears to be minimized for $\mathrm{x}=1.1 \mathrm{y}$.

The calculatedpositions of the band edges of the layers in the strained SL sample are given in Table 1. It is seen that the conduction band offset does not exceed $20 \mathrm{meV}$. For the thermalized electrons at room temperature the influence of the resulting periodical potential should be negligible. Besides, as a result of the conduction-bandline up, the 4-nm barriers for the electrons in the SL are transparent. Thus the changes of electron mobility and spin relaxation rate should be small compared to pure GaAs.

It is seen from Fig. 1 and the data of Table 1 that the strain of the $\mathrm{Al}_{\mathrm{x}} \mathrm{In}_{\mathrm{y}} \mathrm{Ga}_{1-\mathrm{x}-\mathrm{y}} \mathrm{As}$ layers produces barriers for both heavy and light holes, the barrier for the light holes being $75 \mathrm{meV}$ higher, which leads to additional holeminiband splitting favorable for the electron optical orientation.

Table 1. The positions (in eV) of the band edges at room temperature in an $\mathrm{Al}_{0.20} \mathrm{In}_{0.18} \mathrm{Ga}_{0.62} \mathrm{As} / \mathrm{GaAs} \mathrm{SL}$ sample. ${ }^{\mathrm{a}}$

\begin{tabular}{|c|c|c|c|c|c|}
\hline $\mathrm{E}_{\mathrm{vh} 2}$ & $\mathrm{E}_{\mathrm{vl} 2}$ & $\mathrm{E}_{\mathrm{cl}}$ & $\mathrm{E}_{\mathrm{c} 2}$ & $\mathrm{E}_{\mathrm{g} 1}$ & $\mathrm{E}_{\mathrm{g} 2}$ \\
\hline$-0.0566^{\mathrm{b}}$ & -0.138 & 1.423 & 1.445 & 1.422 & 1.501 \\
\hline
\end{tabular}
'1- GaAs, 2- $\mathrm{Al}_{\mathrm{x}} \mathrm{In}_{\mathrm{y}} \mathrm{Ga}_{1-\mathrm{x}-\mathrm{y}}$ As layer.
b Zero energy is at valence-band edge of the GaAs layer.

The choice of the layer thickness is dictated by the need to split the hole minibands. The splitting grows when barriers are broad enough and wells are narrow and deep. Still the thickness of the strained $\mathrm{Al}_{\mathrm{x}} \mathrm{In}_{\mathrm{y}} \mathrm{Ga}_{1-\mathrm{x}-\mathrm{y}} \mathrm{As}$ layer should be less than the critical thickness $h_{c}(y)$. The overall critical thickness for the superlattice with alternating layers of equal thickness can be estimated as $H_{c}=h_{c}(y / 2)$. The thickness of a single $\mathrm{Al}_{\mathrm{x}} \mathrm{In}_{\mathrm{y}} \mathrm{Ga}_{1-\mathrm{x}-\mathrm{y}} \mathrm{As}$ layer was taken to be $4 \mathrm{~nm}$ (to be less than the calculated $h_{c}(y)$ ), and in accordance with $\mathrm{X}$ - ray data the chosen thickness of the SL sample $(0.136 \mu \mathrm{m})$ exceeded $H_{c}$ much less than in the case of a cathode structure with one strained GaAs layer.

A model-dependent calculation of the hole miniband energies yields absolute values of $E_{h h 1}=13 \mathrm{meV}$ and $E_{l h 1}=54 \mathrm{meV}$. For a small conduction-band offset the electronic band can be taken as the average of the conduction-band energies of the contacting layers. Thus the band gap, $E_{g}$, can be estimated as: 


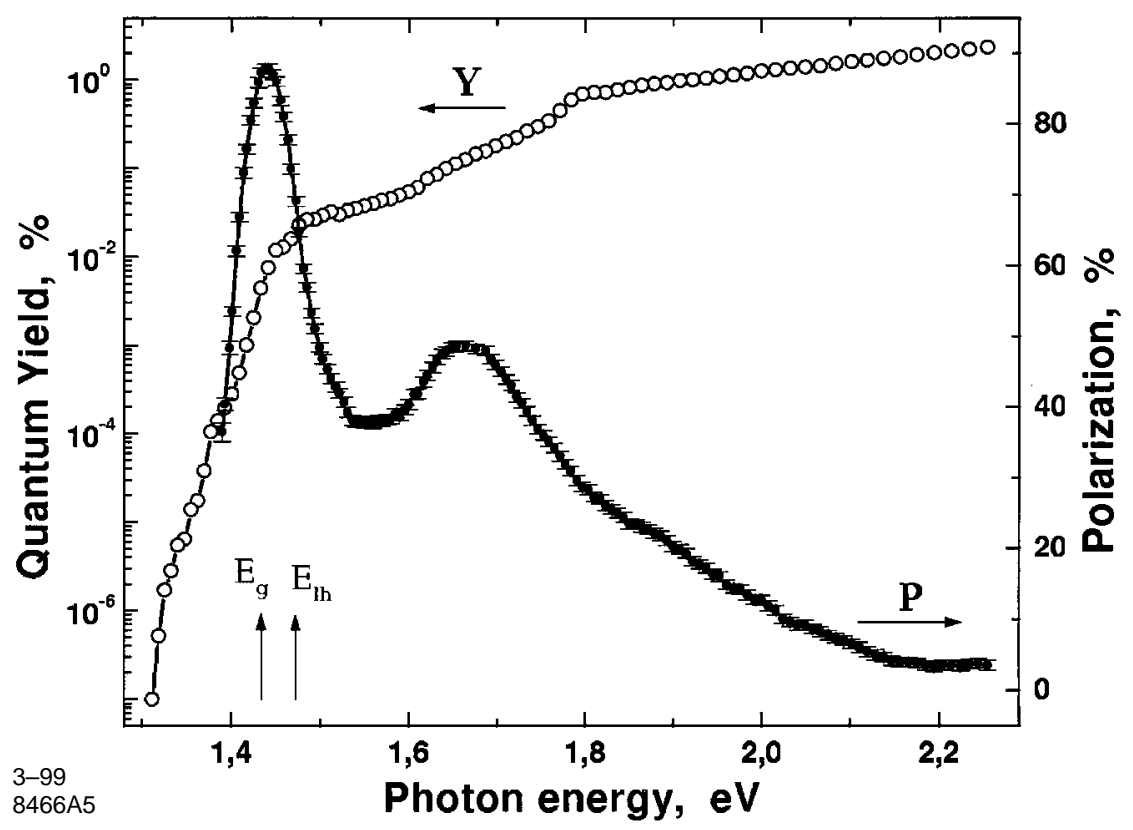

Fig. 2. Electron spin polarization and quantum yield as a function of excitation energy at room temperature for the $\mathrm{Al}_{0.20} \mathrm{In}_{0.18} \mathrm{Ga}_{0.62} \mathrm{As} / \mathrm{GaAs} \mathrm{SL}$ sample (SPTU data). The band gap energy $\mathrm{E}_{\mathrm{g}}$ and the light hole excitation energy $\mathrm{E}_{\mathrm{lh}}$ are indicated by arrows.

$$
E_{g}=E_{g 1}+E_{h h 1}+\left(E_{c 2}-E_{c 1}\right) / 2,
$$

which is in line with experiment.

\section{EXPERIMENTAL RESULTS}

The Mott analysers both at St. Petersburg Technical University (SPTU) and at the Stanford Linear Accelerator Center (SLAC) were used to measure the spin polarization, $P$, of photoelectrons.

In Fig. 2 the polarized emission and quantum yield data measured at SPTU are shown as a function of the optical excitation energy. The maximum polarization obtained was $86 \%$ and the corresponding quantum yield was $7.5 \times 10^{-3} \%$. The yield for the excitation energy at the polarization maximum is sensitive to the activation procedure and the vacuum in the setup. At SLAC, $\mathrm{P}=82.7 \%$ with $Y=9.4 \times 10^{-2} \%$ was obtained for a crystal obtained from the same wafer. For another sample (not shown), in which the time lapse between the two sets of measurements was very short, the polarization results were found to be similar despite the somewhat different techniques of sample preparation, activation, and vacuum. Due to better vacuum conditions in the SLAC apparatus, the values of $Y$ measured there were regularly higher than those measured at SPTU.

The maximum current density that can be extracted from the SL samples similar to the one described above have yet to be determined. Initial measurements at SLAC gave anomalously low values, perhaps because the surfaces were being heat-cleaned at $600{ }^{\circ} \mathrm{C}$ exactly as for the SLC strained-layer cathodes. Other SL structures-all of which were produced in Japan-that have been studied for charge limit have shown normal emission after removal of the As cap at low $\left(\sim 400{ }^{\circ} \mathrm{C}\right)$ temperature $[5,6]$.
There is now strong evidence that the SCL can be overcome by using extremely high dopant density $\left(\geq 4 \times 10^{19} \mathrm{~cm}^{-3}\right)$ in at least the surface layer [6]. This greatly reduces the barrier to holes tunneling to the surface, which are responsible for neutralizing the surface charge. It may also be necessary to increase the negative affinity at the surface by using a large band gap and also possibly a large offset of the conduction miniband. All of these parameters are easily controlled for an MBE-grown SL structure with compensated conduction band as described here.

\section{CONCLUSIONS}

Electron spin polarization as high as $86 \%$ has been obtained at room temperature from strained $\mathrm{Al}_{\mathrm{x}} \mathrm{In}_{\mathrm{y}} \mathrm{Ga}_{1-\mathrm{x}-}$ ${ }_{y} \mathrm{As} / \mathrm{GaAs}$ superlattice with minimal conduction band offset. Modulation doping of the SL provides high polarization and high quantum yield. The position of the polarization maximum can be easily tuned to an excitation wavelength by choice of the SL composition. Further improvement of the emitter parameters can be expected with additional optimization of the SL structure parameters.

\section{REFERENCES}

[1] R.A. Alley et al., Nucl. Instrum. and Meth. A 365 (1995) 1, and references therein.

[2] A.V. Subashiev et al., Phys. Low-Dim. Struct. 1/2 (1999) 1, also issued as preprint SLAC-PUB-8035 (1998).

[3] T. Nakanishi et al., Proc. of Low Energy Polarized Electron Workshop, St. Petersburg (1998) 118; also Polarized Gas Targets and Polarized Beams, AIP Conf. Proc. 421 (1998) 300.

[4] A.V. Subashiev et al., Proc. of Low Energy Polarized Electron Workshop, St. Petersburg (1998) 55, also issued as preprint SLAC-PUB-7995 (1998)

[5] Y. Kurihara et al., Jpn. J. Appl. Phys. 34 (1995) 355.

[6] K. Togawa et al., Nucl. Instrum. and Meth. A 414 (1998) 431. 